\title{
Single-phase gold/palladium catalyst: The nature of synergistic effect
}

\author{
Laura Prati $^{\text {a,*, }}$, Alberto Villa ${ }^{\text {a }}$, Francesca Porta ${ }^{a}$, Di Wang ${ }^{\text {, }}$, Dangsheng Su ${ }^{\text {b }}$ \\ ${ }^{a}$ Dipartimento di Chimica Inorganica Metallorganica e Analitica e INSTM, Centre of Excellence \\ CIMAINA, Universita`di Milano, via Venezian 21, I-20133 Milano, Italy \\ ${ }^{\mathrm{b}}$ Department of Inorganic Chemistry, Fritz Haber Institut der MPG, ELCASS, European Laboratory of \\ Catalysis and Surface Scienc Faradayweg 4-6, D-14195 Berlin, Germany \\ * Corresponding author. Tel.: +39 02503 14357; fax: +39 02503 14405. E-mail address: Laura.Prati@unimi.it (L. Prati).
}

\begin{abstract}
The liquid phase oxidation of glycerol with oxygen has been studied using mono and bimetallic catalysts based on $\mathrm{Au}$ and Pd metals supported on activated carbon, in order to study the effect of the metal on the distribution of the products and on activity of catalysts. It was found that by using bimetallic catalysts $(\mathrm{Au}-\mathrm{Pd})$ a strong synergistic effect was shown. By using a preformed nucleating centre we were able to obtain a single alloyed phase, which allowed us to address the synergistic effect to the presence of alloyed $\mathrm{Au} / \mathrm{Pd}$. The advantage of using this latter catalyst lies not only in the high activity but also in a prolonged catalyst life. Although a partial leaching of palladium and assembling of the particles have been revealed by ICP and HRTEM respectively, activity after 10 re-cycles decreased less than expected (about 10\%).
\end{abstract}

Keywords: Glyceric acid; Liquid-phase oxidation of glycerol; Gold; Palladium; Alloyed gold/palladium; Synergistic effect

\section{Introduction}

Bimetallic catalysts have been received attention as new features can be produced from combination of a lot of metal [1,2]. In particular Au/Pd system has been extensively studied [3-6] as the two metals are miscible in almost all the composition and reactivity of palladium and gold are particularly attractive as in liquid phase oxidation with oxygen, they respectively show a high activity and a high resistance to oxygen poisoning [7].

The synergistic effect between gold and palladiummetals is a well-recognized phenomenon in liquid phase oxidation [2,8-11]. However, segregation or multiple phases have been often evidenced on the same catalyst, thus making the correlation between active specie and activity very difficult and speculative. Therefore, the design of the catalyst represents a compulsory task for the understanding of such multi-metallic catalytic systems.

For gold/palladium system, alloyed, as well as core-shell particle has been prepared [12,13]. For catalytic purposes small, nanometric particles are normally needed affording to highly dispersed system thus precluding method involving thermal treatment. We already experienced preparation of $\mathrm{Au} / \mathrm{Pd}$ systems using sol techniques, which provided a rather good control in particle size and high metal dispersion when supported on activated carbon [9]. However neither coreduction nor subsequent reduction of $\mathrm{Au}(\mathrm{III})$ and Pd(II) salts produced a single-phase colloid, with segregation of Pd always detected after the immobilizing step.

In this study we report on a modified preparation procedure of $\mathrm{Au}-\mathrm{Pd}$ on carbon catalyst aimed to obtain a single-phase system that after careful characterisation, allows to address the relation between nature of the catalyst and its activity. Modification of chemical properties with respect to monometallic systems was expected to improve both activity and durability of the catalyst in liquid phase oxidation of glycerol. This represents an important reaction for the industrial revaluation of this co-product in biodiesel manufacturing [14-16]. It has already been reported that $\mathrm{Pd} / \mathrm{Au}$ nanoparticles supported on activated carbon show a strong synergistic effect $[10,17]$.

\section{Experimental}

Gold of 99.9999 purity in sponge from Fluka, $\mathrm{Na}_{2} \mathrm{PdCl}_{4} \_99.998 \%, \mathrm{NaAuCl}_{4} 2 \mathrm{H}_{2} \mathrm{O} 99.0 \%$ purity from Aldrich and activated carbon from Camel (X40S; SA $=1100-1200 \mathrm{~m}^{2} \mathrm{~g}^{-1} ; \mathrm{pH} 8-9$ )were employed. Before use the carbonwas suspended in $\mathrm{HCl} 6$ Mand left under stirring for $12 \mathrm{~h}$, then was washed several 
times with distilled water by decantation until the $\mathrm{pH}$ of the solution reached value of 6-6.5. At the end the carbon was filtered off and dried for $5-6 \mathrm{~h}$ at $150{ }^{\circ} \mathrm{C}$ in air. The final water content was evaluated to be $<3 \%$.

$\mathrm{NaBH}_{4} 99.0 \%$ purity and polyvinylalcohol 87-89\% hydrolysed (PVA) (average $\mathrm{M}_{\mathrm{w}}$ 13,000-23,000) from Aldrich were used. $\mathrm{NaOH}$ was from Fluka of the highest purity available. Gaseous oxygen and hydrogen from SIAD was $99.99 \%$ pure.

Glycerol (88 wt.\% solution), glyceric acid and all the intermediates were from Fluka. Milli-Q water was used in all the preparations.

\subsection{Catalyst preparations}

Monometallic catalysts were prepared as reported elsewhere by using a preformed metallic sol [9]. Bimetallic catalyst was prepared by using the two-step procedure reported in [18]: $\mathrm{NaAuCl}_{4} \cdot 2 \mathrm{H}_{2} \mathrm{O}(0.072$ mmol) dissolved in $140 \mathrm{ml}$ of $\mathrm{H}_{2} \mathrm{O}$ in the presence of PVA $(2 \%$, w/w, $0.706 \mathrm{ml})$ was reduced by $0.1 \mathrm{M}$ $\mathrm{NaBH}_{4}$. The ruby red $\mathrm{Au}(0)$ sol was immediately formed and immobilised by adding activated carbon under vigorous stirring. The amount of support was calculated as having a final metal loading of 0.73 wt.\%. After $2 \mathrm{~h}$ the slurry was filtered, the catalyst washed thoroughly with distilled water (neutral mother liquors). ICP analyses were performed on the filtrate using a Jobin Yvon JY24 to verify the metal loading on carbon. The $\mathrm{Au} / \mathrm{C}$ was then dispersed in $140 \mathrm{ml}$ of water; $\mathrm{Na}_{2} \mathrm{PdCl}_{4}$ solution (10 wt.\% in $\left.\mathrm{Pd}\right)(0.0386$ $\mathrm{ml})$ and PVA $(2 \%, \mathrm{w} / \mathrm{w})(0.225 \mathrm{ml})$ were added and $\mathrm{H}_{2}$ bubbled $(50 \mathrm{ml} / \mathrm{min})$ under atmospheric pressure and at room temperature for $2 \mathrm{~h}$. After additional $18 \mathrm{~h}$ the slurry was filtered, the catalyst washed thoroughly with distilled water. ICP analyses were performed on the filtrate using a Jobin Yvon JY24 to verify the final Pd metal loading on carbon to be $0.27 \mathrm{wt} . \%$.

\subsection{Characterisations}

(a) The gold and palladium concentrations was checked by ICP analysis of the filtrate or alternatively directly on catalyst after burning off the carbon, on a Jobin Yvon JY24. The water content was determined by drying a sample at $1508 \mathrm{C}$ in air for $5 \mathrm{~h}$.

(b) The SEM images are taken by a Hitachi S4800 scanning electron microscope equipped with cold field emission gun (FEG). Operating voltage varies from $0.1 \mathrm{kV}$ to $30 \mathrm{kV}$. The images shown were taken at 5 $\mathrm{kV}$. EDX spectra were taken by EDX detector in the microscope.

(c) For TEM investigations, the powder samples of the catalysts were ultrasonically dispersed in ethanol and mounted onto a copper grid covered with holey carbon film. A Philips CM200 FEG electron microscope, operating at $200 \mathrm{kV}$ and equipped with a Gatan imaging filter, GIF100, was used for TEM observation. EDX analysis was performed in the same microscope using a DX4 analyzer system (EDAX).

\subsection{Oxidation experiments}

The reactions were carried out in a thermostatted glass reactor $(30 \mathrm{ml})$ provided with an electronically controlled magnetic stirrer connected to a large reservoir $(5000 \mathrm{ml})$ containing oxygen at $3 \mathrm{~atm}$. The oxygen uptake was followed by a mass flow controller connected to a PC through an AC/DC board, plotting a flow/time diagram.

Glycerol $(0.3 \mathrm{M}), \mathrm{NaOH}(\mathrm{NaOH} / \mathrm{glycerol}=4 \mathrm{~mol} / \mathrm{mol})$ and the catalyst $($ glycerol $/ \mathrm{metals}=3000 \mathrm{~mol} / \mathrm{mol})$ were mixed in distilled water. The reactor was pressurised at 3 atm of $\mathrm{O}_{2}$ and thermostatted at $50{ }^{\circ} \mathrm{C}$. After an equilibration time of $10 \mathrm{~min}$, the reaction was started up by stirring and samples were taken every $15 \mathrm{~min}$. Analyses were performed on a Varian 9010 HPLC equipped with a Varian 9050 UV (210 $\mathrm{nm})$ and aWaters R.I. detector in series. An Alltech OA-10308 column (300 mm x $7.8 \mathrm{~mm}$ ) was used with aqueous $\mathrm{H}_{3} \mathrm{PO}_{4} 0.1 \%(\mathrm{w} / \mathrm{w})(0.5 \mathrm{ml} / \mathrm{min})$ as the eluent.

Recycling experiments were carried out by filtering off the catalyst after $0.5 \mathrm{~h}$ reaction. The catalyst was simply washed with distilled water and used again with a fresh solution of glycerol/ $\mathrm{NaOH}$.

\section{Results and discussion}

We used preformed gold particle as nucleation centre for the subsequent deposition of Pd particle; thus we immobilised on carbon a preformed metallic sol of gold and then use this $\mathrm{Au} / \mathrm{C}$ material as the support for Pd deposition producing a bimetallic system 1\% Pd@Au/C. The catalyst was characterized by TEM techniques, including (HR) TEM (Fig. 1) and EDX spectroscopy (Fig. 2). The observations on the morphology and the microstructures concerning both the phase and the composition of the nanoparticles offer the direct evidence of its "single phase" property [18]. The size distribution can be fitted approximately by a log-normal function, implying a coalescence growth mechanism [19]. However the coalescence is limited due to the presence of PVA. This can be evidenced from the fact that only about $2 \%$ particles have size from $10 \mathrm{~nm}$ up to $30 \mathrm{~nm}$. The size distribution is very narrow and most particles 
have the size of 3-4 nm. The ratios of $\mathrm{Pd}$ to $\mathrm{Au}$ are almost unchanged for all the examined single particles, regardless of the particle size. Moreover, the ratio is also similar to that of the overall measurement, indicating that there is no segregation of Pd or Au. In combination with the HRTEM observations, which show uniform structure within each particle, we can confirm that all the particles consist of random $\mathrm{Au}-\mathrm{Pd}$ alloy, with the $\mathrm{Au}-\mathrm{Pd}$ ratio close to 6:4. The lattice parameter of this alloy is between that of $\mathrm{Au}$ and that of Pd. The catalytic data in the selective liquid phase oxidation of glycerol to glycerate $\left(0.3 \mathrm{M}\right.$ water solution, glycerol $/ \mathrm{NaOH}=4 \mathrm{~mol} / \mathrm{mol}$, glycerol $/ \mathrm{metal}=3000, p \mathrm{O}_{2}=3 \mathrm{~atm}, T=50$ ${ }^{\circ} \mathrm{C}$ ) confirmed the big enhancement of activity compared to that obtained with $\mathrm{Au}$ or $\mathrm{Pd}$ monometallic catalysts (Fig. 3). TOFs measurements showed a six fold increase from about $1000 \mathrm{~h}^{-1}$ for monometallic to $6400 \mathrm{~h}^{-1}$ for alloyed bimetallic one (Table 1). Due to the unique presence of single Au6Pd4 alloy in $\mathrm{Au} / \mathrm{Pd}$ bimetallic catalyst, we can therefore conclude that the alloyed phase is responsible for the high synergistic effect observed. Selectivity was also improved with respect to monometallic gold, alloyed catalyst maintaining almost the same selectivity of pure palladium (Table 1).

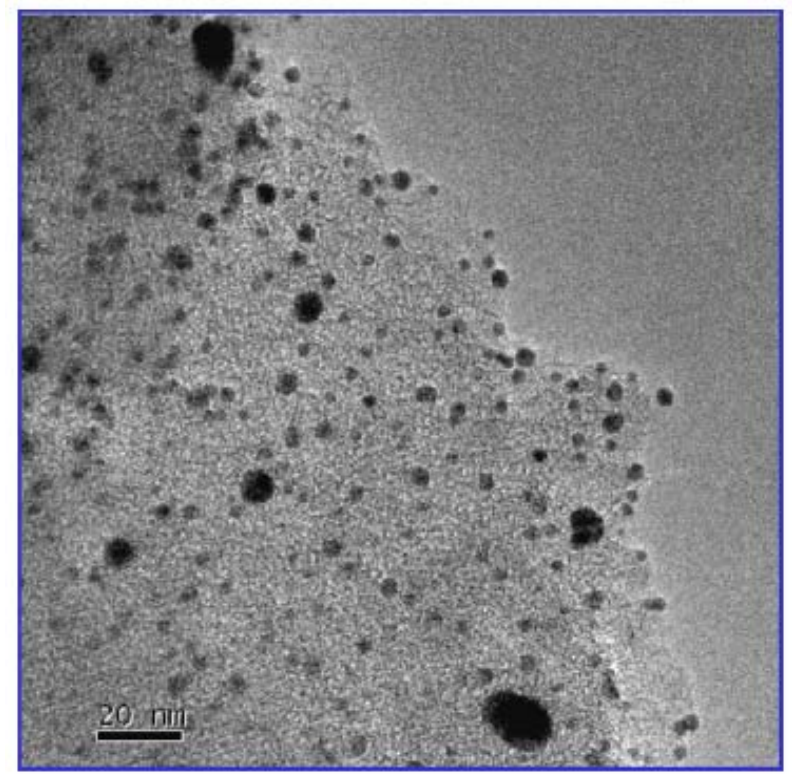

Fig. 1. Overview TEM image of fresh 1 wt.\% Pd@Au/C catalyst.

Note also that $\mathrm{Au}$ and Pd behaved similarly in term of initial activity (TOF) (Table 1), meaning that particle size is comparable according to previous result [20]. However a fundamental difference between gold and palladium could be derived observing conversion profiles (Fig. 3). Palladium catalyst deactivated rapidly, whereas neither gold nor bimetallic alloyed system showed this phenomenon.

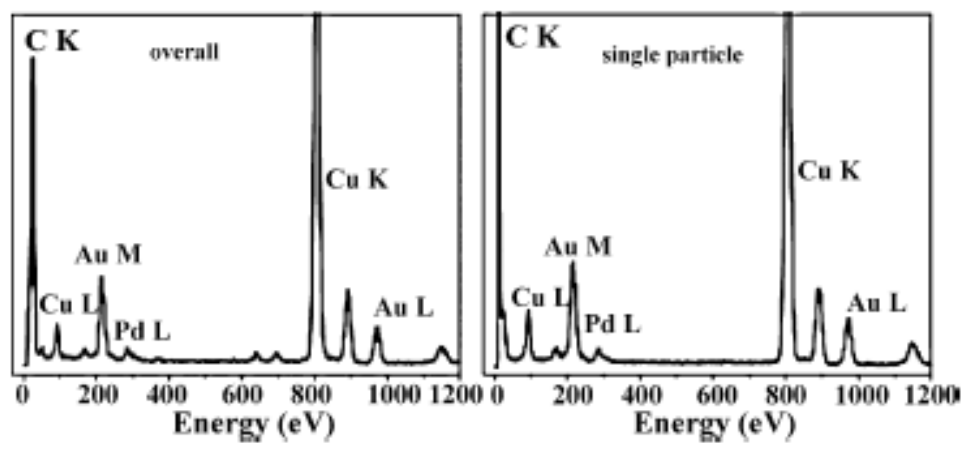

Fig. 2. The overall EDX spectrum (left) and the representative spectra taken from individual single particle (right).

It was suggested that binding energy of oxygen to palladium decreases when palladium atoms are surrounded by gold [21,22]. Moreover, it was found in acetoxylation of ethylene to vinyl acetate that $\mathrm{Au}$ acted as promoter to isolate $\mathrm{Pd}$ monomer sites, preventing undesirable pathways to $\mathrm{CO}, \mathrm{CO}_{2}$ and surface carbon [5]. The same mechanism could be responsible for avoiding deactivation, which is severe for the pure Pd catalyst. 


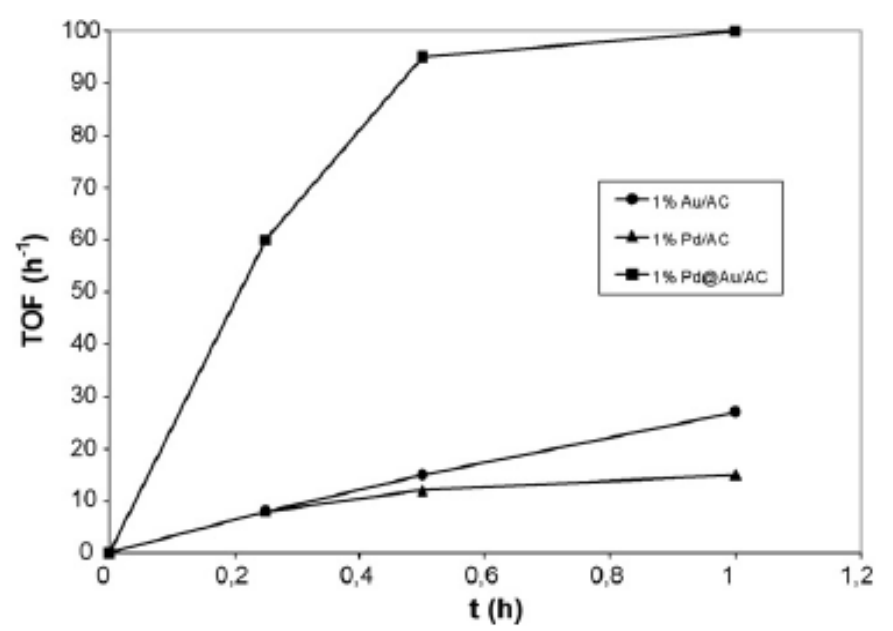

Fig. 3. Selective oxidation of glycerol with mono and bimetallic catalysts

Reaction conditions: water $10 \mathrm{ml}, 0.3 \mathrm{M}$ glycerol, glycerol $/ \mathrm{M}=3000, \mathrm{NaOH} /$ glycerol $=4, T=50{ }^{\circ} \mathrm{C}, p \mathrm{O} 2=3 \mathrm{~atm}$.

However, improved resistance to self-poisoning is not enough to explain the strong synergistic effect observed and also changes in surface coverage of adsorbed species could result in increase specific activity. Modification of lattice parameter suggests change also in surface interatomic distances and therefore in the electronic structure, even though there is no considerable electron transfer between $\mathrm{Pd}$ and $\mathrm{Au}$ [23]. Therefore, the number of Pd or Au monomers on the bimetallic catalyst surfaces is greatly reduced and the highly improved activity could be attributed to the Au-Pd bifunctional sites. Such sites have been determined by HREELS to exist in a model catalyst at the edge of $\mathrm{Au}$ islands on $\operatorname{Pd}\left(\begin{array}{lll}1 & 1 & 1\end{array}\right)$ surface, responsible for CO "linear" adsorption [24]. Improvement in activity could then be considered from a positive combination between electronic and geometric effects [25]. It is important to observe that the balance between these two effects could be also modified by the support.

Table 1

Selective oxidation of glycerol using mono and bimetallic catalysts at $50^{\circ} \mathrm{C}^{\mathrm{a}}$

\begin{tabular}{|c|c|c|c|c|c|}
\hline Catalyst & TOF $\left(h^{-1}\right)^{b}$ & S50 GLY & S50 $(\text { GLY+TART })^{d}$ & S90 GLY & S90 $\sum(\text { GLY+TART })^{d}$ \\
\hline $1 \% \mathrm{Au} / \mathrm{C}$ & 908 & 68 & 73 & 68 & 73 \\
\hline $0.7 \% \mathrm{Au} / \mathrm{C}^{\mathrm{e}}$ & 900 & 68 & 73 & 66 & 73 \\
\hline $1 \% \mathrm{Pd} / \mathrm{C}^{\mathrm{f}}$ & 1000 & $80 \mathrm{f}$ & $83 \mathrm{f}$ & - & - \\
\hline $1 \% \operatorname{Pd} @ \mathrm{Au} / \mathrm{C}$ & 6435 & 77 & 83 & 77 & 83 \\
\hline
\end{tabular}

${ }^{a}$ Reaction conditions: water $10 \mathrm{ml}, 0.3 \mathrm{M}$ glycerol, glycerol $/ \mathrm{M}=3000, \mathrm{NaOH} /$ glycerol $=4, T=50^{\circ} \mathrm{C}, p \mathrm{O}_{2}=3 \mathrm{~atm}$.

${ }^{\mathrm{b}}$ Calculation of TOF $\left(\mathrm{h}^{-1}\right)$ after $0.25 \mathrm{~h}$ of reaction. TOF numbers were calculated on the basis of total loading of metals.

${ }^{c}$ Selectivity to glyceric acid at $50 \%$ and $90 \%$ conversion.

${ }^{\mathrm{d}}$ Selectivity to glyceric + tartronic acids at $50 \%$ and $90 \%$ conversion.

e $\mathrm{Au} / \mathrm{C}$ precursor of $1 \% \mathrm{Pd} @ \mathrm{Au} / \mathrm{C}$.

${ }^{\mathrm{f}}$ This catalyst deactivate before reaching $50 \%$ conversion. Selectivity at $20 \%$ conversion is reported.

Stability of the catalyst under operative conditions was checked by re-using the same catalyst in 11 runs (10 re-cycles). The catalyst was simply filtered off, washed with water and without any other treatment used in a new run using fresh glycerol/ $\mathrm{NaOH}$ solution. Conversions after $30 \mathrm{~min}$ for each run are shown in Fig. 4. We observed an almost stable conversion up to the 4 th run (less than $4 \%$ loss), then a decreasing of about $6 \%(10 \%$ on initial conversion) after other 6 cycles. Part of this decreasing is expected on the basis of normal loosing of catalyst on recycling, especially considering the small amount handled (less than $100 \mathrm{mg}$ ). We carried out Au and Pd checking by ICP on collective solution. The first period (4 runs) evidenced presence of palladium (less than $1 \%$ on the basis of the total) and an increased amount in the second period ( $4 \%$ on the basis of the total). No gold was detected.

The catalyst used (11 runs, 10 re-cycles) was characterized by means of SEM and TEM. According to SEM (Fig. 5) observation metal particles appeared still well dispersed on carbon. However, some aggregation of particles took place in agreement with the TEM observation (Fig. 6) compared to that of fresh catalyst (Fig. 1). However, high resolution TEM image (Fig. 7) of used catalyst revealed that "aggregation" could be better described as "assembling" of metal particles. Each particle appeared to maintain more or less its individuality. This finding together with the relatively small decreasing in activity observed during the catalyst re-cycling seems to indicate that catalytically active surface has not been greatly affected. Moreover, EDX spectra in TEM and SEM indicated that the concentration ratio of $\mathrm{Au}$ to Pd varies largely for different regions and particles, which is very different from the fresh catalyst, 
meaning that leaching of palladium occurred not homogeneously. Thus, although catalytic performances did not differ a lot from initial one, the single-phase nature of fresh catalyst has been lost. Different contribution to catalytic activity from different phases could be expected.

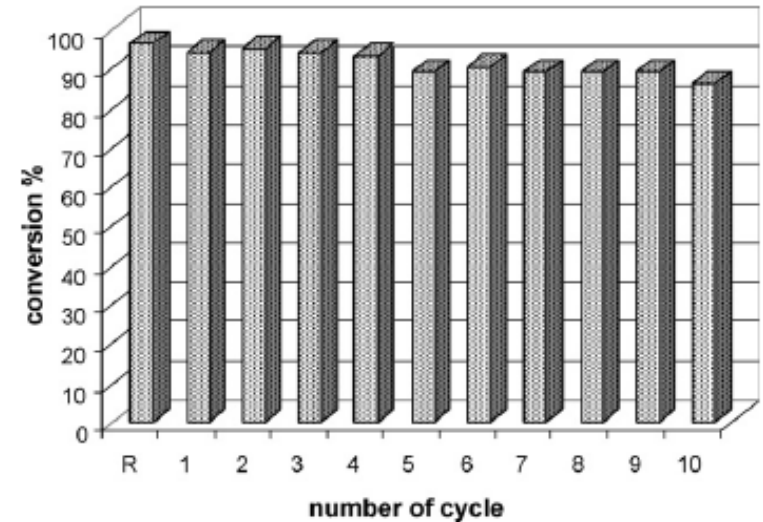

Fig. 4. Recycling experiments of $1 \% \mathrm{Pd} @ \mathrm{Au} / \mathrm{C}$ catalyst in glycerol oxidation.

Reaction conditions: water $30 \mathrm{ml}, 0.3 \mathrm{M}$ glycerol, glycerol $/ \mathrm{M}=3000, \mathrm{NaOH} /$ glycerol $=4, T=50^{\circ} \mathrm{C}, p \mathrm{O}_{2}=3 \mathrm{~atm}$
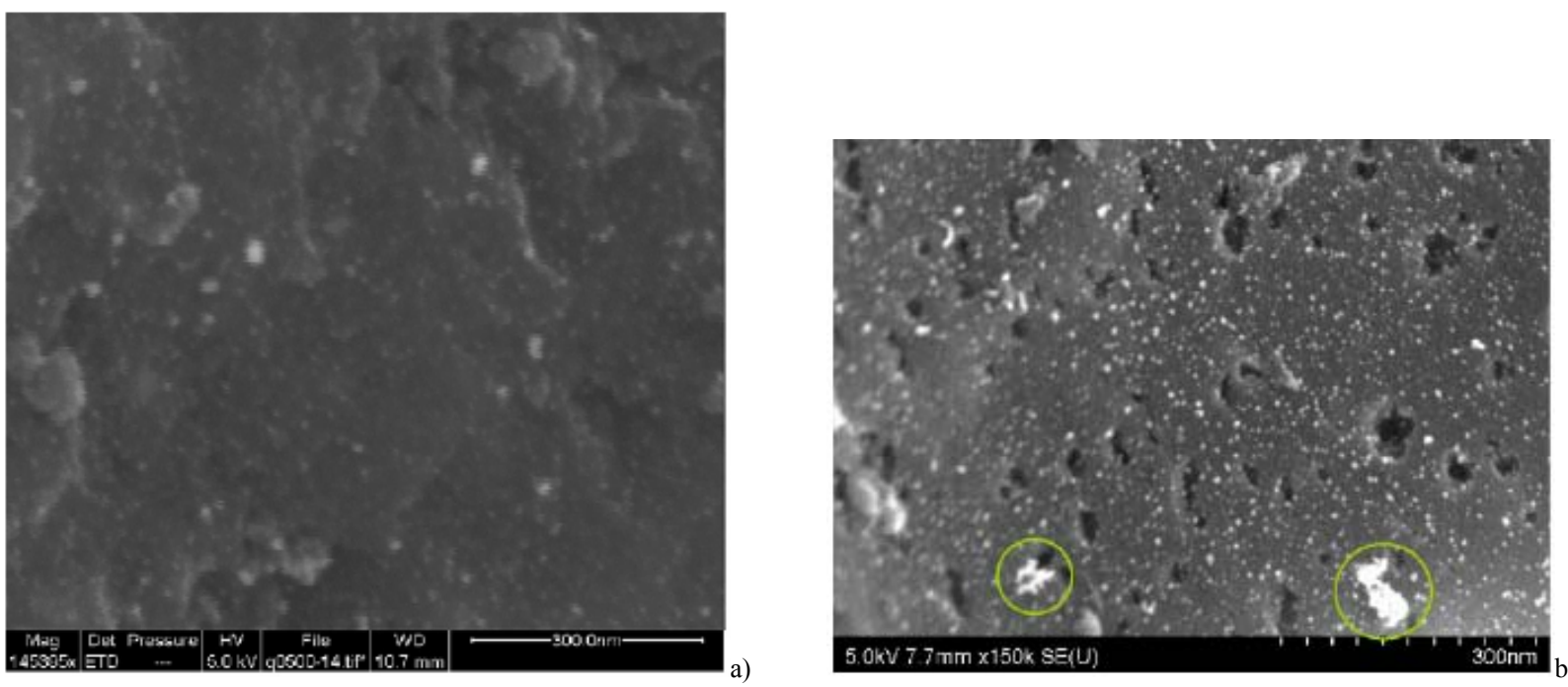

Fig. 5. SEM images for a) fresh and b) used 1\% Pd@Au/C.

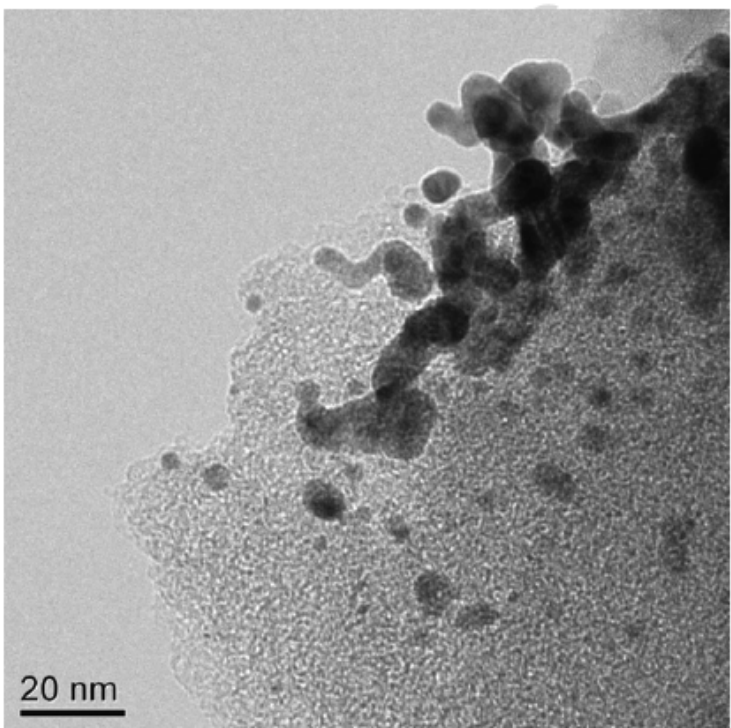

Fig. 6. Particle assembling in 10 re-cycles used catalyst. 

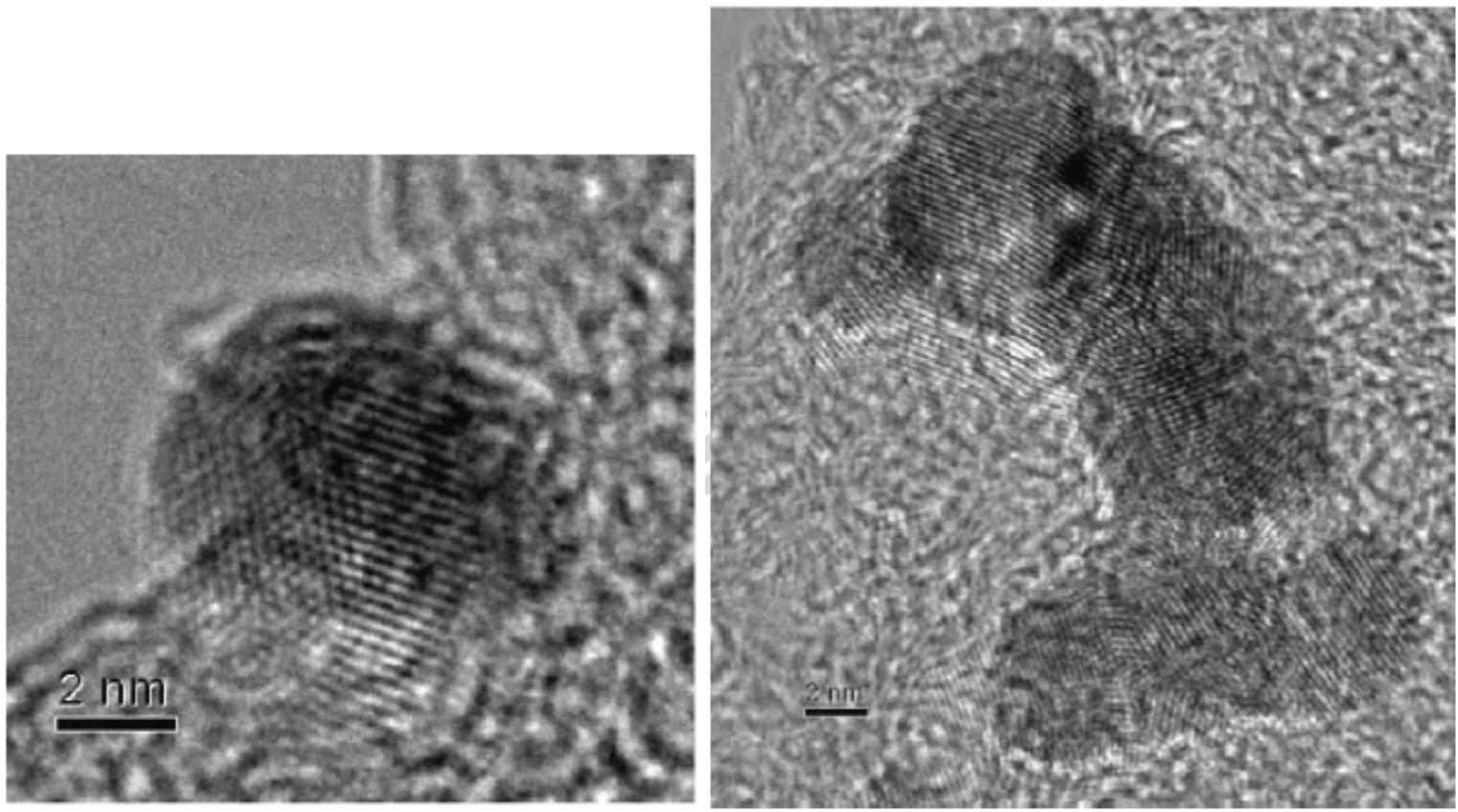

Fig. 7. HRTEM of a single particle and for assembled particles in used 1\% Pd@Au/C.

\section{Conclusions}

Using a two-step synthesis combining colloidal and impregnation techniques, we were able to prepare a bimetallic $\mathrm{Au} / \mathrm{Pd}$ catalyst characterized by the presence of a single Au6Pd4 alloy and small particle size (mean size $3.4 \mathrm{~nm}$ ). This catalyst showed a strong enhancement in activity when it was used in the selective oxidation of glycerol, the enhancement that can be univocally ascribed to the presence of alloy. Both electronic and geometrical effect participated in determining a strong positive synergetic effect between gold and palladium.

Catalyst life appeared also improved despite a small, an inhomogeneous leaching of palladium could be observed. Reconstruction of metal particle has not been evidenced whereas assembling has been observed.

Further studies are needed for establishing contribution of different phases to catalytic activity as well as for avoiding palladium leaching.

\section{References}

[1] Q. Wang, A.E. Ostafin, in: H.S. Nalwa (Ed.), Encyclopedia of Nanoscience and Nanotecnology, vol. 5, American Scientific Pub., California, 2004, pp. 475-503.

[2] J. Schwank, Gold Bull. 2 (1985) 18, and references cited therein.

[3] L. Guczi, Catal. Today 53 (2005) 101, and references cited therein.

[4] A.E. Hahelin-Weaver, J.F. Weaver, G.B. Hoflund, G.N. Salaita, J. Alloys Comp. 393 (2005) 93.

[5] Y. Robach, M. Abel, L. Porte, Surf. Sci. 526 (2003) 248.

[6] M. Chen, D. Kumar, C.W. Yi, D.W. Goodman, Science 310 (2005) 291.

[7] L. Prati, M. Rossi, J. Catal. 176 (1998) 552.

[8] D.I. Enache, J.K. Edwards, P. Landon, B. Solsona-Espriu, A.F. Carley, A.A. Herzing, M. Watanabe, C.J. Kiely, D.W. Knight, G.J. Hutchings, Science 11 (2006) 362.

[9] J.K. Edwards, B. Solsona-Espriu, P. Landon, A.F. Carley, A.A. Herzing, C.J. Kiely, G.J. Hutchings, J. Catal. $236(2005) 69$.

[10] C.L. Bianchi, P. Canton, N. Dimitratos, F. Porta, L. Prati, Catal. Today 102 (2005) 203.

[11] N. Dimitratos, F. Porta, L. Prati, A. Villa, Catal. Lett. 9 (2005) 181.

[12] Y. Tan, Y. Li, D. Zhu, in: H.S. Nalwa (Ed.), Encyclopedia of Nanoscience and Nanotecnology, vol. 8, American Scientific Pub., California, 2004, pp. 9-40.

[13] V.I. Parvulescu, V. Parvulescu, U. Endurushat, G. Filoti, F.E. Wagner, C. Kubel, R. Richards, Chem. Eur. J. 12 (2006) 2343.

[14] S. Carrettin, P. McMorn, P. Johnston, K. Griffin, G.J. Hutchings, Chem. Commun. 696 (2002).

[15] L. Prati, F. Porta, J. Catal. 224 (2004) 397.

[16] J. Van Gerpen, Fuel Processing Tech. 86 (2005) 1097.

[17] N. Dimitratos, F. Porta, L. Prati, A Villa, Catal. Lett. 9 (2005) 181.

[18] D.Wang, A. Villa, F. Porta, D. Su, L. Prati, Chem. Commun. (2006) 1956.

[19] C.G. Granqvist, R.A. Buhrman, J. Catal. 42 (1976) 477.

[20] N. Dimitratos, J.A. Lopez-Sanchez, D. Lennon, F. Porta, L. Prati, A. Villa, Catal. Lett. 108 (2006) 147. 
[21] Y.L. Lam, J. Criado, M. Boudart, New. J. Chem. 1 (1977) 461.

[22] D.L. Weissman-Wenocur, W.E. Spicer, Surf. Sci. 133 (1893) 499.

[23] L.A. Kibler, A.M. El-Aziz, R. Hoyer, D.M. Kolb, Angew. Chem. Int. Ed.

[24] B. Gleich, M. Ruff, R.J. Behm, Surf. Sci. 386 (1997) 48.

[25] A. Grob, Top. Catal. 37 (2006) 29. 